\title{
Quantitative CT-based bone strength parameters for the prediction of novel spinal implant stability using resonance frequency analysis: a cadaveric study involving experimental micro-CT and clinical multislice CT
}

Daisuke Nakashima ${ }^{1}$, Ken Ishii ${ }^{1,2}$, Yuji Nishiwaki ${ }^{3}$, Hiromasa Kawana ${ }^{4}$, Masahiro Jinzaki ${ }^{5}$, Morio Matsumoto ${ }^{1}$ Masaya Nakamura ${ }^{1}$ and Takeo Nagura ${ }^{1,6^{*}}$ (D)

\begin{abstract}
Background: To predict conventional test forces (peak torque and pull-out force) and a new test force (implant stability quotient [ISQ] value of a spinal pedicle screw) from computed tomography (CT) parameters, including micro-architectural parameters, using high-resolution micro-CT and clinical multislice CT (MSCT) in human cadaveric vertebrae.
\end{abstract}

Methods: Micro-CT scans before/after screw insertion $(n=68)$ and MSCT scans before screw insertion $(n=58)$ of human cadaveric vertebrae were assessed for conventional test forces and ISQ value. Three-dimensional volume position adjustment between pre-insertion micro-CT and MSCT scans and post-insertion scans (micro$\mathrm{CT}$ ) was performed to extract the volume of the cancellous bone surrounding the pedicle screw. The following volume bone mineral density and micro-architectural parameters were calculated: bone volume fraction, bone surface density (bone surface/total volume (BS/TV)), trabecular thickness, trabecular separation, trabecular number, structure model index, and number of nodes (branch points) of the cancellous bone network/total volume (NNd/TV) using Spearman's rank correlation coefficient with Bonferroni correction.

Results: Conventional test forces showed the strongest correlation with BS/TV: peak torque, $\rho=0.811, p=4.96 \times$ $10^{-17}$ (micro-CT) and $\rho=0.730, p=7.87 \times 10^{-11}$ (MSCT); pull-out force, $\rho=0.730, p=1.64 \times 10^{-12}$ (micro-CT) and $\rho=0.693, p=1.64 \times 10^{-9}(\mathrm{MSCT})$. ISQ value showed the strongest correlation with NNd/TV: $\rho=0.607, p=4.01 \times 10^{-8}$ (micro-CT) and $\rho=0.515, p=3.52 \times 10^{-5}$ (MSCT).

Conclusions: Test forces, including the ISQ value, can be predicted using micro-CT and MSCT parameters. This is useful for establishing a preoperative fixation strength evaluation system.

Keywords: Bone density, Pedicle screws, Resonance frequency analysis, Torque, X-ray (microtomography)

\footnotetext{
* Correspondence: nagura@keio.jp

'Department of Orthopedic surgery, Keio University School of Medicine, 35

Shinanomachi, Shinjuku, Tokyo, Japan

${ }^{6}$ Department of Clinical Biomechanics, Keio University School of Medicine,

Shinjuku, Tokyo, Japan

Full list of author information is available at the end of the article
} 


\section{Key points}

- Implant stability quotient (ISQ) value is a new test force that reflects in vivo stress.

- ISQ value and conventional test forces of spinal implant stability are different.

- These test forces could be predicted using both microCT and clinical multislice CT.

- Bone surface density is the most effective in predicting conventional test forces.

- Cancellous bone network/total volume branch points are suitable in predicting ISQ value.

\section{Background}

Spinal instrumentation surgeries are popular, and an increasing number of surgeries is being performed annually $[1,2]$. Implant stability is important in spinal instrumentation surgeries, as it ensures secure fixation of the implant to the bone and prevents implant fixation failure $[3,4]$. In particular, in the field of spinal surgery, the rate of postoperative pedicle screw loosening has been reported to be up to $12 \%$ [5, 6]. Galbusera et al. [7] reported a screw loosening incidence of up to $60 \%$ among patients with osteoporosis. Pedicle screw loosening is one of the major indications for revision after spinal surgery [8]. An appropriate initial test force is important to achieve good surgical results and prevent screw loosening. To overcome these issues, both the prediction of test force using preoperative images and establishment of an intraoperative test force evaluation system are required.

With regard to the prediction of test force using preoperative images, some reports have demonstrated a correlation between the test force and bone mineral density (BMD) of the osteoporotic vertebra assessed using two-dimensional dual-energy $\mathrm{x}$-ray absorptiometry (DEXA), which is one of the preoperative imaging modalities $[9,10]$. Although computed tomography (CT) is also frequently used for planning spinal surgery, it is not yet commonly performed in clinical practice to predict the test force of a pedicle screw using imaging data.

The term bone strength indicates resistance to bone destruction and was defined by the National Institutes of Health Consensus 2000 Development Panel on Osteoporosis Prevention, Diagnosis, and Therapy [11]. BMD is frequently used as an indirect measure of bone strength and accounts for approximately $70 \%$ of bone strength. The remaining $30 \%$ of bone strength is considered to involve bone quality, which has been described as the flexibility and soundness of the bone structure. In $\mathrm{CT}$, bone quality can be represented by digitising the three-dimensional (3D) structure of the bone with patterns such as honeycomb-, plate-, and beam-shaped patterns. These structures are represented by micro-architectural parameters [12]. Many recent studies have reported that BMD assessed using DEXA alone may not be sufficient to determine the strength of cancellous bone and that trabecular architecture may be an important predictor of bone strength and fracture risk [13, 14].

With regard to the establishment of an intraoperative test force evaluation system, pull-out force $[3,15,16]$ and insertion torque $[3,17]$ are the test force measures generally used to evaluate screw stability. Pull-out force is measured destructively through laboratory testing and is defined as the maximum axial force required to pull a screw out from the bone [18]. Additionally, it is not possible to measure insertion torque more than once after screw fixation. Thus, pull-out force and insertion torque are not used as intraoperative measures.

We have previously reported [19] on a system involving resonance frequency analysis (RFA) [20-22] that can be used for the intraoperative evaluation of a pedicle screw (Osstell apparatus). With this system, the dedicated parameter implant stability quotient (ISQ) can be assessed, and its value ranges from 0 (lowest stability) to 100 (highest stability) [23]. An ISQ value of 0 represents a resonance frequency of approximately $3,000 \mathrm{~Hz}$, and a value of 100 represents a resonance frequency of approximately $8,000 \mathrm{~Hz}$ [24]. This non-invasive and repeatable technique might indicate multidirectional test force $[19,23]$, which differs from insertion torque and pull-out force that reflect only axial force [19].

The present study aimed to predict conventional test forces (peak torque and pull-out force) and a new test force (ISQ value of a spinal pedicle screw) from CT parameters, including micro-architectural parameters, using experimental high-resolution micro-CT and clinical multislice CT (MSCT) in human cadaveric vertebrae.

\section{Methods \\ Spinal screws}

Monoaxial pedicle screws (Catalog no. CMS05135, Kyocera Medical Corporation, Kyoto, Japan) measuring $45 \mathrm{~mm}$ in length, with an outer threaded diameter of 5.5 $\mathrm{mm}$, an inner threaded diameter of $3.8 \mathrm{~mm}$ (start point), and of $4.6 \mathrm{~mm}$ (end point), made of titanium alloy (Ti-6Al-4 V[ELI], American Society for Testing and Materials [ASTM] F136) were used in this study. To measure the ISQ value with the Osstell ISQ ${ }^{\circ}$ system (Osstell Integration Diagnostics, Gothenburg, Sweden), two neodymium magnets (Magfine Corporation, Miyagi, Japan) were attached to the head of the pedicle screw as previously reported [19].

\section{Spinal dissection and treatment before the experiment}

For the experimental use of fresh non-frozen human cadavers, written informed consent was obtained from each donor according to the ethical guidelines of our institution 
(approval number: 20070026). Dissection was performed as soon as possible post-mortem. All experiments were approved by the Ethics Committee (approval number: 20150385). Nine human thoracolumbar spinal sections (Th8-L5) were used in this study (mean donor age, 76 years; age range, $72-86$ years; seven female and two male cadavers). The intervertebral disks and ligaments were dissected from the vertebrae. Vertebrae that showed spinal fusion $(n=4)$ or were damaged during preparation $(n=6)$ were excluded. The remaining vertebrae were visually inspected and diagnosed using micro-CT images by two spinal surgeons (11 and 27 years of experience), and vertebrae showing fractures or spinal metastases were excluded $(n=3)$. The specimens were individually wrapped in Ziploc polyethylene bags (S.C. Johnson \& Son, Incorporated, Racine, WI) for micro-CT imaging [17].

\section{Micro-CT and MSCT imaging before pedicle screw insertion} After the scan of a BMD phantom (RATOC, Tokyo, Japan), the vertebrae were scanned using a micro-CT system (R_mCT2 FX, Rigaku Corporation, Tokyo,
Japan) with the following settings: $x$-ray voltage, 90 $\mathrm{kVp}$; tube current, $160 \mu \mathrm{A}$; exposure time, $120 \mathrm{~s}$, continuous (non-stepping) rotation. For the BMD phantom and each vertebra, a stack of 400 cross-sectional slices, corresponding to a total height of $57 \mathrm{~mm}$, was reconstructed with a slice-to-slice distance of 1 pixel $(144 \mu \mathrm{m})$. The scan data of the BMD phantom were used to convert the CT number (Hounsfield units) to $\operatorname{BMD}\left(\mathrm{mg} / \mathrm{cm}^{3}\right)$.

After micro-CT, MSCT was performed. The vertebrae were placed in an in-house rounded rectangle-shaped tank (Fig. 1a, b) that simulated the human body, and MSCT imaging was performed with a BMD phantom (B-MAS200, Fujirebio, Tokyo, Japan) (Fig. 1c, d) using an MSCT system (Discovery CT750, GE Healthcare, Chicago, IL) using a standard protocol (for the phantom, $120 \mathrm{kVp}, 250 \mathrm{~mA}$, voxel size $683 \times 683 \times 5,000 \mu \mathrm{m}$, field of view $350 \times 350$ $\mathrm{mm}$; for the vertebra: $120 \mathrm{kVp}, 250 \mathrm{~mA}$, voxel size $293 \times$ $293 \times 625 \mu \mathrm{m}$, field of view $150 \times 150 \mathrm{~mm}$ ) (Fig. 1d). The scan data of the BMD phantom were used to convert the CT number to BMD $\left(\mathrm{mg} / \mathrm{cm}^{3}\right)$ [25].
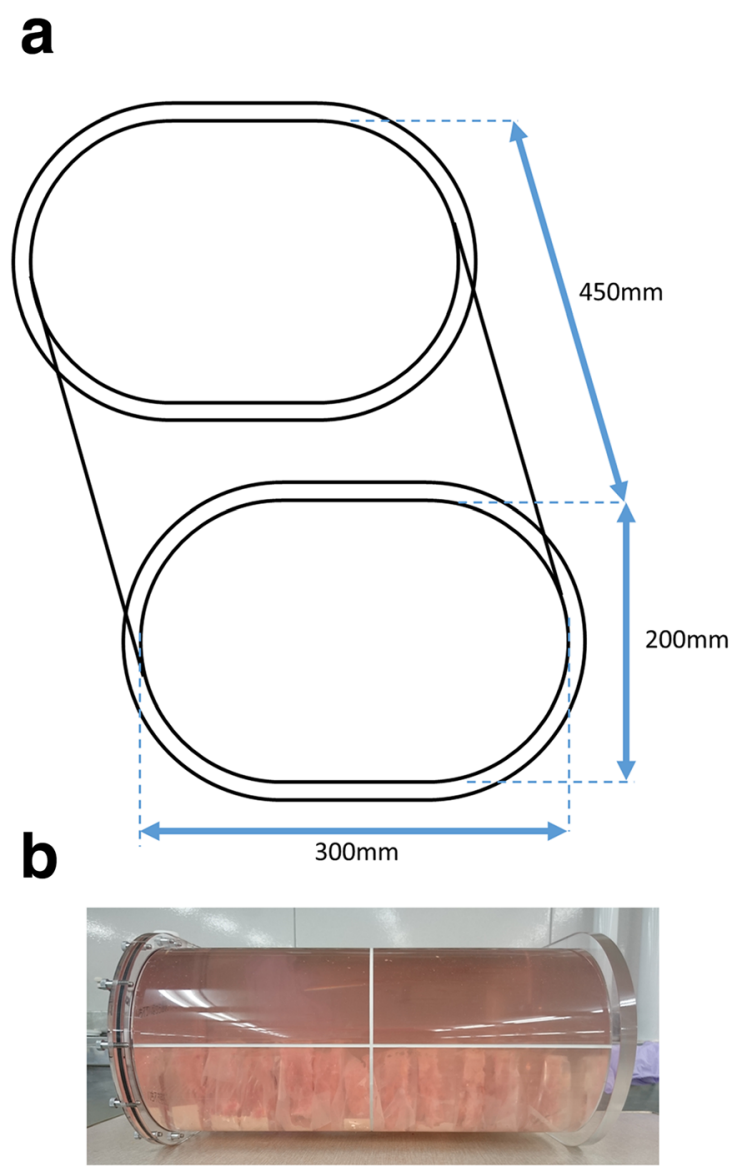

\section{C}
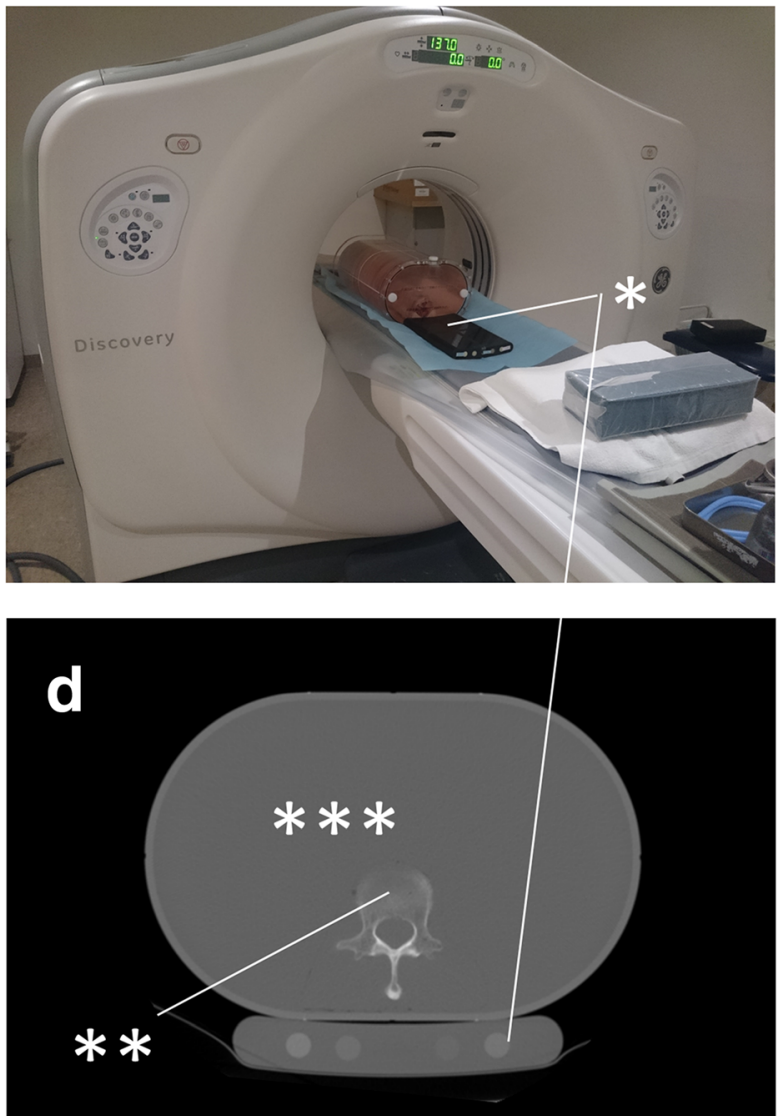

Fig. 1 Multislice computed tomography. a Drawing of the in-house round rectangle-shaped tank. b Vertebrae in the tank. c Photograph of the tank and the phantom $\left(^{*}\right)$ in the scanner. $\mathbf{d}$ Axial image showing the phantom $(*)$, one vertebra $(* *)$, and the surrounding water for mimicking a living body $\left(^{* * *}\right)$ 


\section{Fixation strength experiment \\ Pedicle screw insertion}

Pedicle screw insertion was performed by a spinal surgeon. A pilot hole was drilled in the vertebra using an awl and a $20-\mathrm{mm}$ probe without a tap. The pedicle screw was inserted $40 \mathrm{~mm}$.

\section{Insertion torque measurement}

The digital torque gauge HTGA-5 N (Imada Company Limited, Aichi, Japan) was used to measure the insertion torque (peak torque $[19,26,27]$ ) at $40-\mathrm{mm}$ insertion. The specifications of this torque gauge were as follows: accuracy $\pm 0.5 \%$ (full scale) \pm 1 digit and sampling rate $2,000 \mathrm{data} / \mathrm{s}$. The insertion torque (Newton meter, $\mathrm{N} \mathrm{m}$ ) is a moment force, and it increases progressively as the screw advances in the vertebra. This insertion torque peaks before the screw head comes in contact with the vertebra, which is defined as peak torque. This torque is experienced as the test force of the pedicle screw by the surgeon (Fig. 2a) [19, 26].

\section{Resonance frequency analysis}

It was conducted using a specific device (Osstell Integration Diagnostics, Gothenburg, Sweden) without screw contact after completion of the pedicle screw insertion as reported previously (Fig. 2b) [19]. Materials were not held by a fixture during measurement and were placed on a normal laboratory table instead. The pedicle screw was vibrated with a micromagnetic wave, which generated inertial forces owing to the mass of the magnets in a plane perpendicular to the axis of the screw.

\section{Micro-CT imaging after pedicle screw insertion}

Micro-CT imaging was performed again for the vertebrae with the pedicle screws using the same protocol as that before screw insertion (Fig. 3b).

\section{Pull-out force measurement}

The pull-out force measurement was performed according to ASTM-F543-07 testing standards [28]. The vertebrae were placed on a specially fabricated fixture with a self-position adjustment function to ensure vertical pullout alignment. Then, they were held in the appropriate position on a base plate. The maximum pull-out force was measured using AG-IS $10 \mathrm{kN}$ (Shimadzu Corporation, Kyoto, Japan; testing speed $5 \mathrm{~mm} / \mathrm{min}$ ) [28]. Strength was continuously recorded in $0.1-\mathrm{mm}$ increments until its peak (Fig. 2c).

\section{Volume BMD and micro-architectural parameters of the vertebrae}

Imaging analysis was performed using dedicated software (TRI/3D-BON, RATOC, Tokyo, Japan) to calculate volume $\mathrm{BMD}$ and the micro-architectural parameters of the vertebrae. First, 3D volume position adjustment between pre-insertion (both micro-CT and MSCT) (Fig. 3a) and post-insertion (micro-CT) (Fig. 3b, c) images was performed, and the volume of the cancellous bone where the pedicle screw was present was extracted (Fig. 3d, e). The cutoff value was $120 \mathrm{mg} / \mathrm{cm}^{3}$. The cortex was extracted before calculating the 3D volume position adjustment. Volume BMD and microarchitectural parameters were calculated. The following

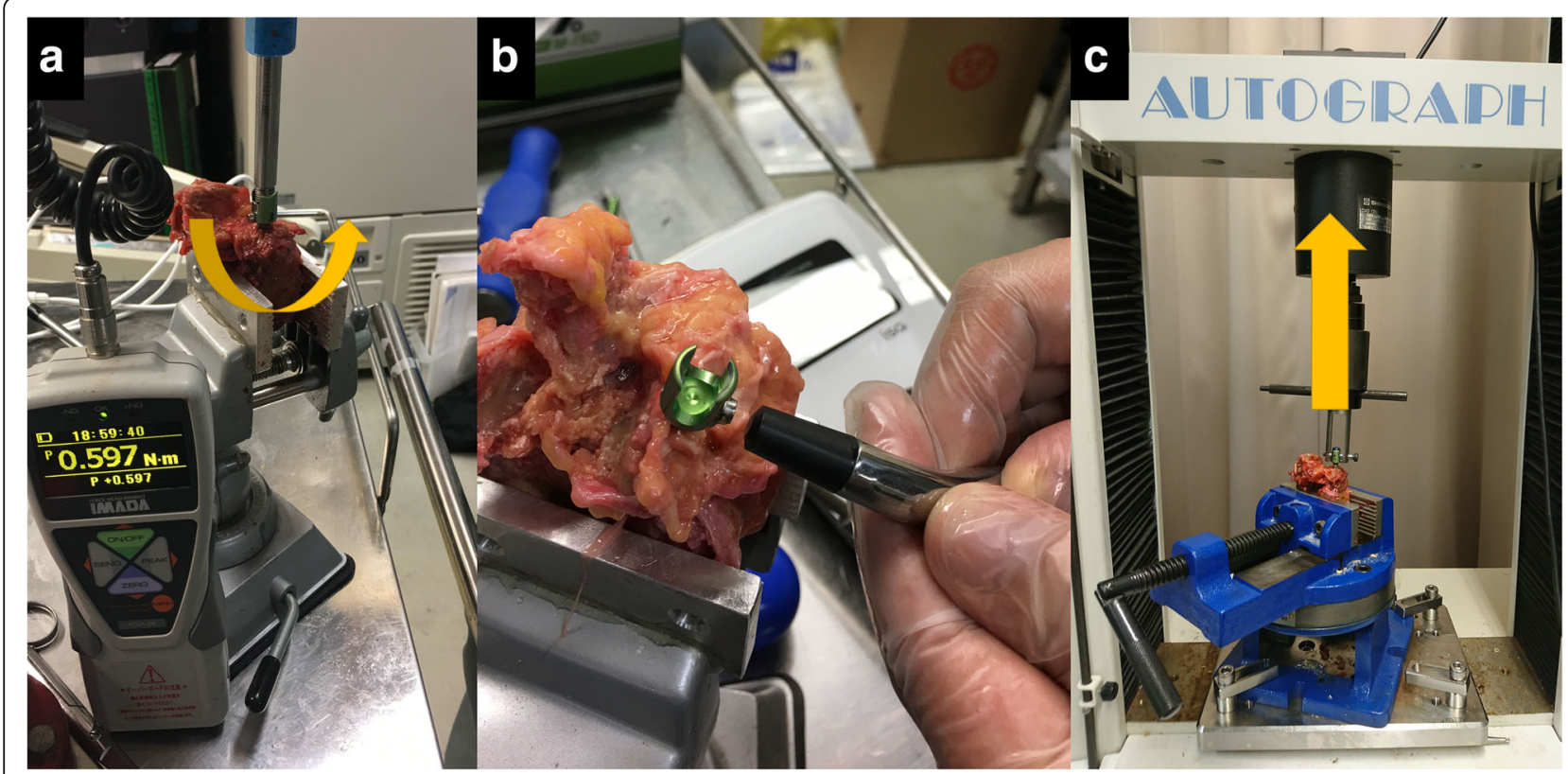

Fig. 2 Test force measurement. a Peak torque measurement. b Implant stability quotient value measurement. c Pull-out force measurement 


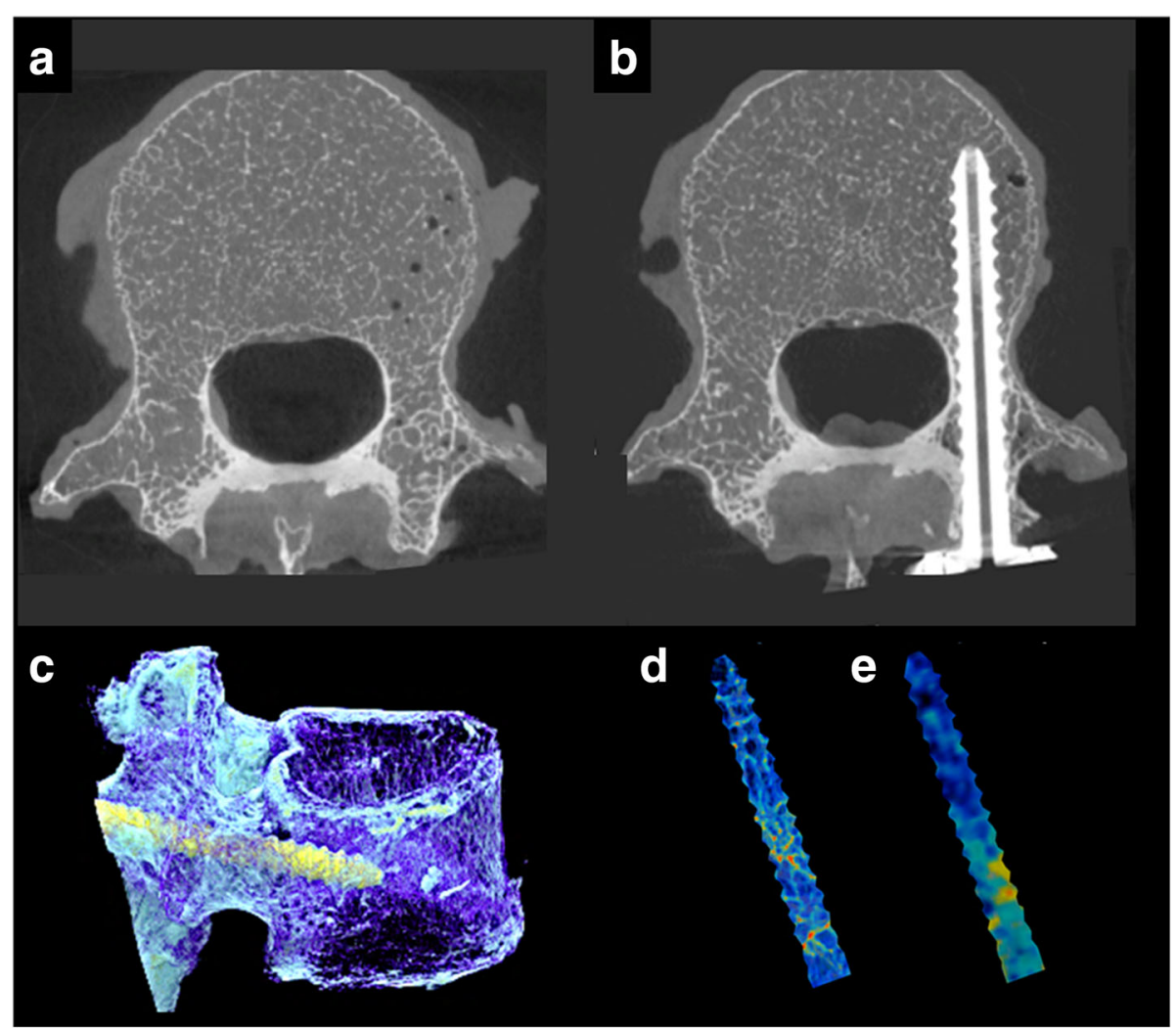

Fig. 3 Micro-CT and MSCT imaging. a Micro-CT axial image of a vertebra. b Micro-CT axial image of a vertebra with a screw. c Three-dimensional micro-CT reconstruction of a vertebra with a screw. Cancellous bone area of the pedicle screw extracted by three-dimensional volume position adjustment between the pre- and post-insertion images: micro-CT (d) and MSCT (e)

micro-architectural parameters were assessed: bone volume fraction (bone volume/total volume: BV/TV, $\%$ ), bone surface density (bone surface/total volume (BS/TV), $\left.\mathrm{mm}^{-1}\right)$, trabecular thickness $(\mathrm{mm})$, trabecular separation $(\mathrm{mm})$, trabecular number $\left(\mathrm{mm}^{-1}\right)$, structure model index (SMI), and number of nodes per volume $\left(\mathrm{NNd} / \mathrm{TV}, \mathrm{mm}^{-3}\right)[17,29-31] . \mathrm{SMI}$ is a measure for the relative number of rod- and plate-like trabecular bone structures, with values ranging from 0 (ideal plate-like structure) to 3 (ideal rod-like structure), values in between representing a mixture of plates and rods [32]. In recent years, SMI has frequently been used to investigate the relationship between $\mathrm{CT}$ and implant fixation strength in research $[17,32]$. NNd/TV, unlike SMI, indicated the complexity of trabecular bone structure from the viewpoint of the number of bonding points of the trabecular beam structure.

\section{Statistical analysis}

The three measures (peak torque, pull-out force, and ISQ value) and parameters of both micro-CT and MSCT were obtained. First, Spearman's rank moment correlation coefficient $(\rho)$ with Bonferroni correction was used to evaluate the relationship among the three measures. The significance level was set at $p=0.0166$. Second, the same analysis was performed to evaluate the relationship between the three measures and the parameters of both micro-CT and MSCT. The significance level was set at $p=0.00625$. All statistical analyses were performed using SPSS Statistics software version 24 (International Business Machines Corporation, Armonk, NY).

\section{Results}

A total of 90 vertebrae were initially included. However, 13 vertebrae met the abovementioned exclusion criteria and 9 vertebrae were excluded due to pedicle destruction during screw insertion. Thus, 68 vertebrae were successfully examined with all assessments, except MSCT imaging parameter assessments. MSCT imaging parameter assessments could not be performed in 10 vertebrae owing to insufficient resolution. Therefore, 58 vertebrae were examined in the MSCT study. Table 1 summarises the three measures and the parameters of CT imaging.

\section{Correlations among the three test forces}

Peak torque showed a strong positive correlation with pull-out force $\left(\rho=0.822, p=8.25 \times 10^{-18}\right)$ (Fig. 4a). The 
Table 1 Summary of the three test forces and parameters of the vertebrae on micro-CT and MSCT

\begin{tabular}{|c|c|c|}
\hline & Measure unit & $\begin{array}{l}\text { Mean (standard } \\
\text { deviation) }\end{array}$ \\
\hline \multicolumn{3}{|l|}{ Test forces $(n=68)$} \\
\hline Peak torque & $\mathrm{Nm}$ & $678(246)$ \\
\hline Pull-out force & $\mathrm{N}$ & $357(130)$ \\
\hline Implant stability quotient value & Arbitrary units & $36.5(7.90)$ \\
\hline \multicolumn{3}{|l|}{ Micro-CT parameters $(n=68)$} \\
\hline Volume bone mineral density & $\mathrm{mg} / \mathrm{cm}^{3}$ & $69.6(30.5)$ \\
\hline Bone volume/total volume & - & $29.6(10.7)$ \\
\hline Bone surface/total volume & $\mathrm{mm}^{-1}$ & $1.80(0.350)$ \\
\hline Trabecular thickness & $\mu \mathrm{m}$ & $328(73.0)$ \\
\hline Trabecular separation & $\mu \mathrm{m}$ & $863(328)$ \\
\hline Trabecular number & $\mathrm{mm}^{-1}$ & $0.876(0.170)$ \\
\hline Structure model index & - & $1.86(0.534)$ \\
\hline Node number/total volume & $\mathrm{mm}^{-3}$ & $0.647(0.159)$ \\
\hline \multicolumn{3}{|l|}{ Multislice CT parameters $(n=58)$} \\
\hline Volume bone mineral density & $\mathrm{mg} / \mathrm{cm}^{3}$ & $183(28.1)$ \\
\hline Bone volume/total volume & - & $91.1(10.8)$ \\
\hline Bone surface/total volume & $\mathrm{mm}^{-1}$ & $4.16(0.903)$ \\
\hline Trabecular thickness & $\mu \mathrm{m}$ & $780(252)$ \\
\hline Trabecular separation & $\mu \mathrm{m}$ & $81.0(14.2)$ \\
\hline Trabecular number & $\mathrm{mm}^{-1}$ & $1.28(0.416)$ \\
\hline Node number/total volume & $\mathrm{mm}^{-3}$ & $0.764(0.0230)$ \\
\hline
\end{tabular}

CT computed tomography, $N$ Newton

ISQ value showed weak positive correlations with peak torque ( $\rho=0.242, p=0.00425)$ (Fig. $4 \mathrm{~b}$ ) and pull-out force ( $\rho=0.296, p=0.0146)$ (Fig. 4c). These results are similar to those obtained in a model bone study [19].

\section{Micro-CT study: correlations between the three measures} and imaging parameters

Table 2 summarises the correlations between the three test forces and CT imaging parameters. Both conventional test forces (peak torque and pull-out force) showed the strongest correlations with $\mathrm{BS} / \mathrm{TV}$ (peak torque: $\rho=0.811$, $p=4.96 \times 10^{-17}$; pull-out force: $\rho=0.730, p=1.64 \times 10^{-12}$ ) (Fig. 5a, b). Additionally, both test forces showed lower but significant correlations with volume BMD and micro-architectural parameters, except BS/TV ( $\rho$ ranging from -0.588 to 0.582 ). Interestingly, the ISQ value showed the strongest correlation with $\mathrm{NNd} / \mathrm{TV}(\rho=0.607$, $p=4.01 \times 10^{-8}$ ), unlike the conventional test forces (Fig. 5c). However, it was not significantly correlated with volume $\mathrm{BMD}, \mathrm{BV} / \mathrm{TV}, \mathrm{BS} / \mathrm{TV}$, trabecular thickness, trabecular separation, trabecular number, and SMI.

\section{MSCT study: correlations between the three measures and imaging parameters}

Both conventional test forces (peak torque and pull-out force) showed the strongest correlations with BS/TV (peak torque: $\rho=0.730, p=7.87 \times 10^{-11}$; pull-out force: $\rho=0.693 p=1.64 \times 10^{-9}$ ) (Fig. $\left.5 \mathrm{~d}, \mathrm{e}\right)$. Additionally, both test forces showed lower but significant correlations with volume BMD, trabecular thickness, and trabecular number ( $\rho$ ranging from -0.489 to 0.479 ). The ISQ value showed the strongest moderate correlation with $\mathrm{NNd} /$ TV $\left(\rho=0.515, p=3.52 \times 10^{-5}\right)$, similar to the finding in the micro-CT study (Fig. 5f). The ISQ value showed a lower but significant correlation with BS/TV $(\rho=0.267$, $p=0.0427)$. However, it was not significantly correlated with volume BMD, BV/TV, BS/TV, trabecular thickness, trabecular separation, and trabecular number. SMI could not be calculated because of the lower resolution of MSCT images compared with that of micro-CT images.

\section{Discussion}

The present study found that peak torque and pull-out force had the strongest correlations with BS/TV and that the ISQ value had the strongest correlation with $\mathrm{NNd} /$ TV on micro-CT and MSCT. Our findings indicate that test forces, including the ISQ value, can be predicted by CT parameters on both micro-CT and MSCT. Many
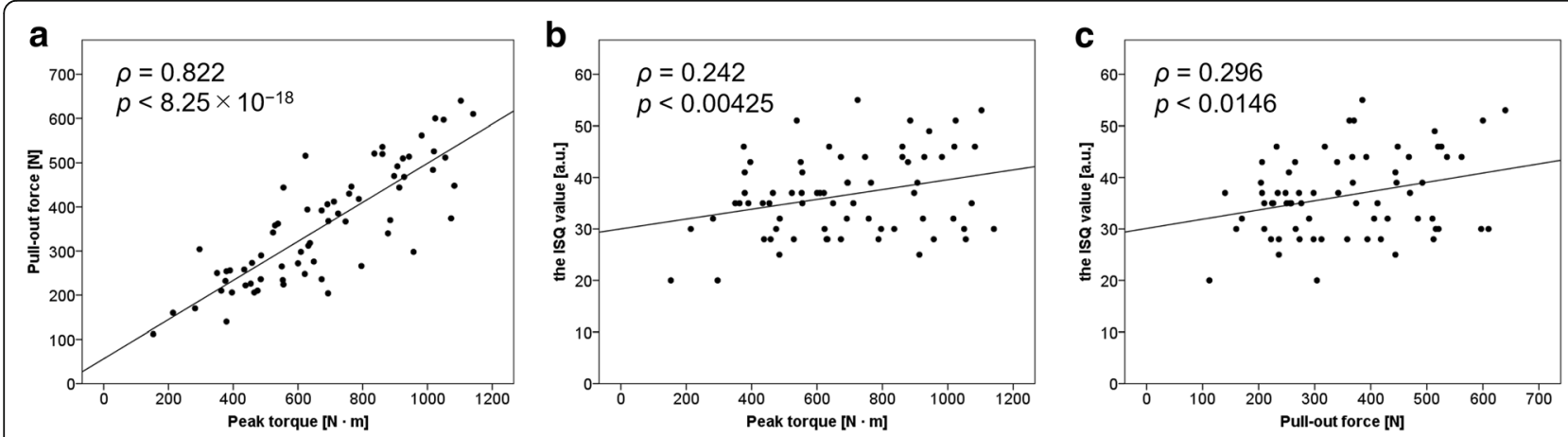

Fig. 4 Scatter plots and best fit lines of linear regression among the three test forces. a Peak torque versus pull-out force. $\mathbf{b}$ Peak torque versus implant stability quotient (ISQ) value. c Pull-out force versus implant stability quotient value. N, Newton; Nm Newton meter, a.u. arbitrary units 
Table 2 Correlation coefficients and $p$ values for the three test forces versus $C T$ parameters on micro-CT and multislice $C T$

\begin{tabular}{|c|c|c|c|c|c|}
\hline & & \multicolumn{2}{|c|}{ Micro-CT $(n=68)$} & \multicolumn{2}{|c|}{ Multislice CT $(n=58)$} \\
\hline & & $\rho$ & $p$ & $\rho$ & $p$ \\
\hline \multirow[t]{8}{*}{ Peak torque versus } & Volume bone mineral density & 0.520 & $5.46 \times 10^{-6 *}$ & 0.463 & $2.50 \times 10^{-4 *}$ \\
\hline & Bone volume/total volume & 0.550 & $1.19 \times 10^{-6 *}$ & 0.334 & 0.0103 \\
\hline & Bone surface/total volume & 0.811 & $4.96 \times 10^{-17 *}$ & 0.730 & $7.87 \times 10^{-11 *}$ \\
\hline & Trabecular thickness & 0.427 & $2.79 \times 10^{-4 *}$ & 0.479 & $1.41 \times 10^{-4 *}$ \\
\hline & Trabecular separation & -0.588 & $1.38 \times 10^{-7 *}$ & -0.267 & 0.0424 \\
\hline & Trabecular number & 0.582 & $1.95 \times 10^{-7 *}$ & -0.489 & $9.66 \times 10^{-5 *}$ \\
\hline & Structure model index & -0.437 & $1.96 \times 10^{-4 *}$ & N.A. & N.A. \\
\hline & Node number/total volume & 0.428 & $2.71 \times 10^{-4 *}$ & -0.069 & 0.608 \\
\hline \multirow[t]{8}{*}{ Pull-out versus } & Volume bone mineral density & 0.453 & $1.06 \times 10^{-4 *}$ & 0.414 & $0.00124^{*}$ \\
\hline & Bone volume/total volume & 0.467 & $5.86 \times 10^{-5 *}$ & 0.320 & 0.0143 \\
\hline & Bone surface/total volume & 0.730 & $1.64 \times 10^{-12 *}$ & 0.693 & $1.64 \times 10^{-9 *}$ \\
\hline & Trabecular thickness & 0.361 & $0.00250^{*}$ & 0.416 & $0.00118^{*}$ \\
\hline & Trabecular separation & -0.519 & $5.68 \times 10^{-6 *}$ & -0.272 & 0.0391 \\
\hline & Trabecular number & 0.529 & $3.54 \times 10^{-6 *}$ & -0.411 & $0.00137^{*}$ \\
\hline & Structure model index & -0.360 & $0.00253^{*}$ & N.A. & N.A. \\
\hline & Node number/total volume & 0.483 & $3.02 \times 10^{-5 *}$ & -0.108 & 0.421 \\
\hline \multirow[t]{8}{*}{ Implant stability quotient value versus } & Volume bone mineral density & 0.0722 & 0.558 & -0.103 & 0.444 \\
\hline & Bone volume/total volume & 0.0666 & 0.589 & -0.0760 & 0.571 \\
\hline & Bone surface/total volume & 0.237 & 0.0521 & 0.201 & 0.130 \\
\hline & Trabecular thickness & -0.0542 & 0.661 & -0.106 & 0.427 \\
\hline & Trabecular separation & -0.158 & 0.197 & 0.0770 & 0.566 \\
\hline & Trabecular number & 0.264 & 0.0299 & 0.0830 & 0.536 \\
\hline & Structure model index & -0.0134 & 0.914 & N.A. & N.A. \\
\hline & Node number/total volume & 0.607 & $4.01 \times 10^{-8 *}$ & 0.515 & $3.52 \times 10^{-5 *}$ \\
\hline
\end{tabular}

$C T$ computed tomography, N.A. not available

*Signifiant $p$ value

trials have evaluated the stability of orthopedic implants (i.e., insertion torque and pull-out force) $[9,15,17,33-$ 35]. To our knowledge, this is the first study to predict these test forces with micro-architectural parameters in the field of spinal surgery.

A strong relationship was found between peak torque and pull-out force (Fig. 4a), suggesting that peak torque, which is close to the operator's hand, may be similar to pull-out force, which reflects axial load [18]. Siddiqui et al. [36] reported a strong correlation between the subjective assessment of screw hold and pull-out force. A similar result was obtained in the present study. In this study, RFA was used to evaluate pedicle screw stability. RFA involving the Osstell ISQ system has been used to evaluate dental implant stability [23]. Our research group [19] reported that RFA in spinal surgery reflects multidirectional load, which represents in vivo physiological loading conditions unlike conventional test forces (insertion torque and pull-out force), in a model bone study. In the present study using cadaveric vertebrae, the correlation of RFA with conventional test forces was low (Fig. 4b, c), similar to the finding in the previous report [19].

With regard to volume BMD and micro-architectural parameters on micro-CT, conventional test forces showed significant correlations with all parameters (Table 2). Particularly, BS/TV showed the strongest correlations with peak torque and pull-out force (Fig. 5a, b). The microarchitectural parameters used were microscopic measurements of the cancellous bone structure mainly obtained from micro-CT, and they are currently used in preclinical research $[4,17,37,38]$. Variations in micro-architectural parameters have been reported to affect the stability of an implant according to laboratory failure testing (push-in force, pull-out force, and plateau torque) [17, 37-39].

The significant correlations of volume BMD, BV/TV, and SMI with the two conventional test forces observed in this study are in agreement with those reported in previous studies using a cadaveric femoral head [37, 38]. SMI is a measure of the relative number of rod- and plate-like trabecular structures, with values ranging from 

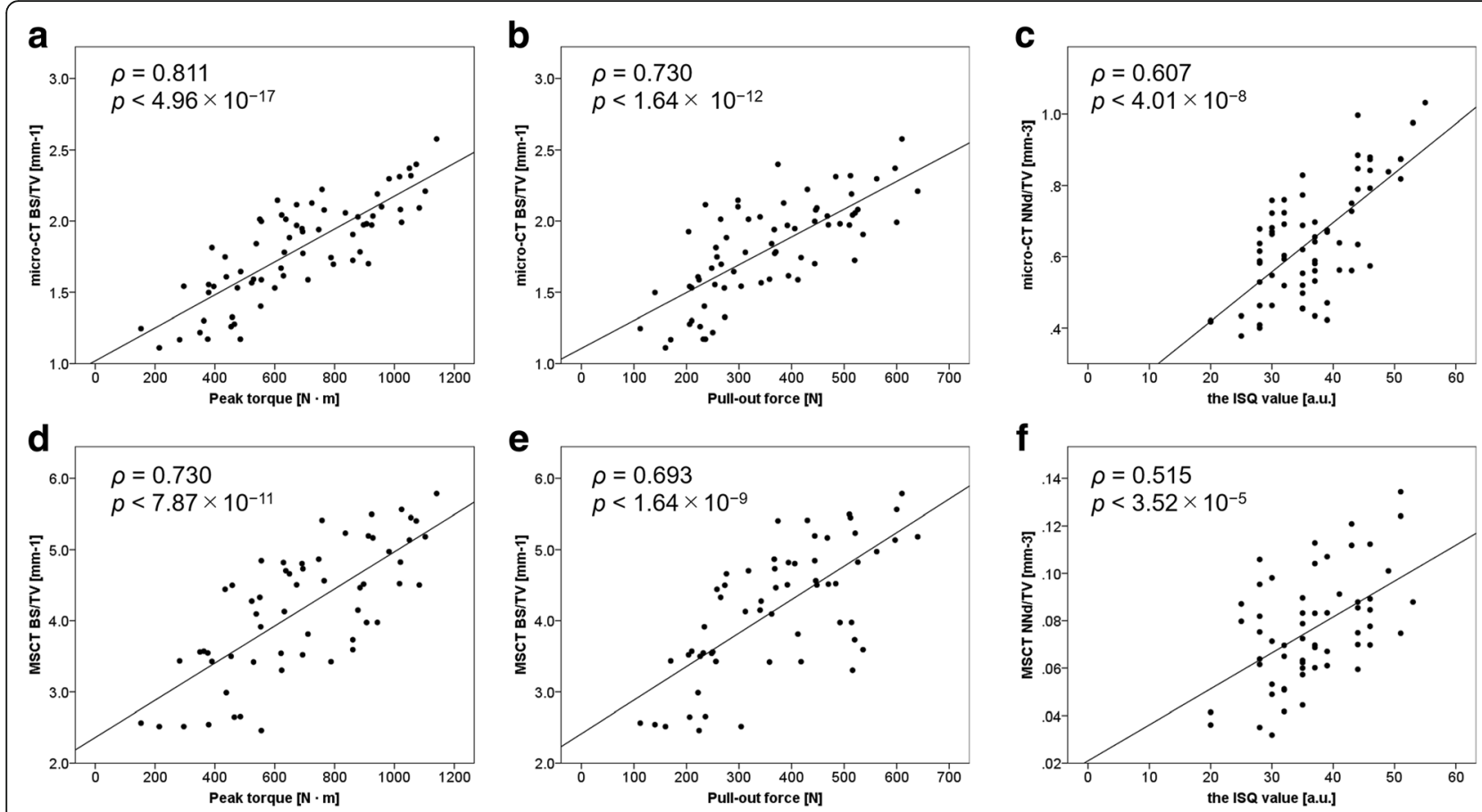

Fig. 5 Scatter plots and best fit lines of linear regression between test forces and micro-architectural parameters (bone surface density) or node number (number of branch points of the cancellous bone network/total volume) on micro- $C T$ and MSCT. a Peak torque versus bone surface density (bone surface $[\mathrm{BS}] /$ total volume [TV]) on micro-CT. b Pull-out force versus BS/TV on micro-CT. c Implant stability quotient (ISQ) value versus node number (NNd)/TV on micro-CT. $\mathbf{d}$ Peak torque versus BS/TV on MSCT. e Pull-out force versus BS/TV on MSCT. $\mathbf{f}$ ISQ value versus NNd/TV on MSCT. N Newton, $\mathrm{Nm}$ Newton meter, a.u. arbitrary units

0 (plate-like structure) to 3 (rod-like structure), with values between 0 and 3 representing a mixture of plates and rods $[31,32,40]$. These results indicate that a platelike bone structure with high BMD or BV exhibits high test forces, as reported in a previous study [17]. Among the volume BMD and micro-architectural parameters in this study, BS/TV was the parameter that best predicted the two test forces by itself. The BS/TV value rises when the bone quality improves with high BMD.

On the other hand, the aforementioned parameters did not show similar regression coefficients for the ISQ value. $\mathrm{NNd} / \mathrm{TV}$, which is the number of branch points of the cancellous bone network/total volume of the measurement region [29], showed the strongest regression coefficient for the ISQ value (Fig. $5 \mathrm{c}$ ). $\mathrm{NNd} / \mathrm{TV}$ is the parameter mainly used for node-strut analysis [29, 41] to evaluate trabecular connectivity. NNd/TV represents the complexity of bone structure (the characteristic of the beam structure, i.e., bone quality), whereas BS/ $\mathrm{TV}$ represents the bone surface area that is close to BMD. Generally, this parameter is used to evaluate the effects of drugs on osteoporosis [29, 30]. The finding indicates that bone quality (trabecular connectivity) has the best correlation with the ISQ value. The correlation coefficients between conventional test forces and BS/TV and that between the ISQ value and $\mathrm{NNd} / \mathrm{TV}$ were lower but sufficient in the MSCT study when compared to the findings in the micro-CT study (Fig. $5 \mathrm{~d}-\mathrm{f}$ ).

In recent years, micro-architectural parameters from MSCT imaging have been reported [42]. In many MSCT studies, cadaveric investigations were performed using MSCT with all soft tissues removed and exposed to air [43]. Under such a condition, no surrounding tissue that can affect MSCT imaging is present, and this imaging condition is much better than the clinical imaging condition of a living body. In order to replicate the situation noted in a living human, we placed the vertebrae in a tank simulating the human body and performed MSCT imaging (Fig. 1a-d). As sufficient correlation was obtained even with examination under strict and disadvantageous circumstances in this study, we believe that the prediction of test forces from preclinical images is sufficiently possible even with the clinical MSCT images. In the future, the correlation coefficients could be improved through the development of high-resolution clinical CT. The present study has some limitations. First, we did not consider the influence of the firmness of the cortical bone of the pedicle itself on the test forces. Currently, a device capable of substantially and non-invasively measuring the bone strength itself is absent. Second, micro-CT imaging was performed on excised specimens in an in vitro setting. Therefore, it did 
not account for the variability of tissues surrounding the bones among the different cadavers. Third, although the specimens were placed in a simulated body, MSCT was performed in an in vitro setting. Fourth, we did not perform a DEXA evaluation of BMD, which remains the most used tool in clinical practice. Therefore, a correlation between DEXA parameters (e.g., BMD, trabecular bone score) might be of further interest. Fifth, the vertebrae with screws were not scanned using MSCT. Although micro-CT alone is sufficient for screw position confirmation, it is more desirable to use the same resolution image for the $3 \mathrm{D}$ volume position adjustment. Nevertheless, the results of this study will be useful in clinical research with the same equipment in the future.

In conclusion, we showed that both micro-CT and MSCT parameters affect the test forces of the pedicle screw (not only conventional test forces but also the ISQ value). Therefore, if their values are evaluated preoperatively, they can be used in the prediction of initial test forces and in preoperative planning (e.g., fixed vertebrae number and implant selection).

\section{Abbreviations \\ 3D: Three-dimensional; ASTM: American Society for Testing and Materials; BMD: Bone mineral density; BS: Bone surface; BV: Bone volume; CT: Computed tomography; DEXA: Dual-energy x-ray absorptiometry; ISQ: Implant stability quotient; RFA: Resonance frequency analysis; SMI: Structure model index; TV: Total volume}

\section{Availability of data and materials}

The datasets used and analysed during the current study are available from the corresponding author on reasonable request.

\section{Funding}

This study has received funding by Japan Agency for Medical Research and Development (Award Number: JP17Im0203004j0001) and Japan Society for the Promotion of Science (Award Number: 16H03174 and 17 J00880).

\section{Acknowledgements}

The authors are grateful to Dr. Nobuaki Imanishi, Mr. Akihisa Yamazaki, Mr. Fumiya Nakada, Mr. Masaki Atarashi, Dr. Ryogo Huruhata, Dr. Keita Kajikawa, Dr. Ken Ninomiya, Dr. Takuto Nozaki, and Dr. Hiroyuki Seki for the spinal dissection and technical assistance in $C T$. The authors acknowledge the contribution of Dr. Naoki Takano for his technical advice and thank the Collaborative Research Resources, School of Medicine, Keio University, for the $\mu \mathrm{CT}$ and technical assistance. The authors also thank the Clinical Anatomy Lab, School of Medicine, Keio University, for the spinal dissection. The authors would like to thank Enago (www.enago.jp) for the English language review.

\section{Authors' contributions}

DN initiated the concept of this study and was a major contributor in writing the manuscript. Kl performed the pull-out test and torque test. YN conducted the statistical study. HK conducted the resonance frequency analysis. MJ conducted the CT-related analysis. MM and MN performed the entire experiment. TN conducted all the research assistance, supervision, and validation. All authors have read and approved the final manuscript.

\section{Ethics approval and consent to participate}

For the experimental use of fresh non-frozen human cadavers, written informed consent was obtained from each donor according to the ethical guidelines of Keio University School of Medicine (approval number: 20070026 and 20150385)

\section{Consent for publication}

Not applicable

\section{Competing interests}

The authors declare that they have no competing interests.

\section{Publisher's Note}

Springer Nature remains neutral with regard to jurisdictional claims in published maps and institutional affiliations.

\section{Author details}

${ }^{1}$ Department of Orthopedic surgery, Keio University School of Medicine, 35 Shinanomachi, Shinjuku, Tokyo, Japan. ${ }^{2}$ Department of Orthopedic surgery, International University of Health and Welfare School of Medicine, Narita, Chiba, Japan. ${ }^{3}$ Department of Environmental and Occupational Health, School of Medicine, Toho University, Tokyo, Japan. ${ }^{4}$ Department of Dentistry and Oral Surgery, Keio University School of Medicine, Shinjuku, Tokyo, Japan. ${ }^{5}$ Department of Radiology, Keio University School of Medicine, Shinjuku, Tokyo, Japan. ${ }^{6}$ Department of Clinical Biomechanics, Keio University School of Medicine, Shinjuku, Tokyo, Japan.

Received: 5 October 2018 Accepted: 20 November 2018

Published online: 22 January 2019

\section{References}

1. Deyo RA, Gray DT, Kreuter W, Mirza S, Martin BI (2005) United States trends in lumbar fusion surgery for degenerative conditions. Spine (Phila Pa 1976) 30:1441-1445 discussion 1446-1447

2. Weinstein JN, Lurie JD, Olson PR, Bronner KK, Fisher ES (2006) United States' trends and regional variations in lumbar spine surgery: 1992-2003. Spine (Phila Pa 1976) 31:2707-2714

3. Kwok AW, Finkelstein JA, Woodside T, Hearn TC, Hu RW (1996) Insertional torque and pull-out strengths of conical and cylindrical pedicle screws in cadaveric bone. Spine (Phila Pa 1976) 21:2429-2434

4. Mueller TL, van Lenthe GH, Stauber M, Gratzke C, Eckstein F, Müller R (2009) Regional, age and gender differences in architectural measures of bone quality and their correlation to bone mechanical competence in the human radius of an elderly population. Bone 45:882-891

5. Bredow J, Boese CK, Werner CM et al (2016) Predictive validity of preoperative $\mathrm{CT}$ scans and the risk of pedicle screw loosening in spinal surgery. Arch Orthop Trauma Surg 136:1063-1067

6. Wittenberg RH, Lee KS, Shea M, White AA 3rd, Hayes WC (1993) Effect of screw diameter, insertion technique, and bone cement augmentation of pedicular screw fixation strength. Clin Orthop Relat Res 296:278-287

7. Galbusera F, Volkheimer D, Reitmaier S, Berger-Roscher N, Kienle A, Wilke HJ (2015) Pedicle screw loosening: a clinically relevant complication? Eur Spine J 24:1005-1016

8. Kocak T, Cakir B, Reichel H, Mattes T (2010) Screw loosening after posterior dynamic stabilization--review of the literature. Acta Chir Orthop Traumatol Cech 77:134-139

9. Halvorson TL, Kelley LA, Thomas KA, Whitecloud TS 3rd, Cook SD (1994) Effects of bone mineral density on pedicle screw fixation. Spine (Phila Pa 1976) 19:2415-2420

10. Soshi S, Shiba R, Kondo H, Murota K (1991) An experimental study on transpedicular screw fixation in relation to osteoporosis of the lumbar spine. Spine (Phila Pa 1976) 16:1335-1341

11. NIH Consensus Development Panel on Osteoporosis Prevention, Diagnosis, and Therapy (2001) Osteoporosis prevention, diagnosis, and therapy. JAMA 285:785-795

12. Bouxsein ML, Boyd SK, Christiansen BA, Guldberg RE, Jepsen KJ, Müller R (2010) Guidelines for assessment of bone microstructure in rodents using micro-computed tomography. J Bone Miner Res 25: 1468-1486

13. Topolinski T, Mazurkiewicz A, Jung S, Cichański A, Nowicki K (2012) Microarchitecture parameters describe bone structure and its strength better than BMD. ScientificWorldJournal 2012:502781

14. Jiang $Y$, Zhao J, Augat $P$ et al (1998) Trabecular bone mineral and calculated structure of human bone specimens scanned by peripheral quantitative computed tomography: relation to biomechanical properties. J Bone Miner Res 13:1783-1790

15. Cakir E, Eyuboglu EE, Yazar U, Gazioglu G, Guvercin AR, Baykal S (2014) May "Dubel" be a solution for pullout problem of the pedicle screws at osteoporotic spine? Turk Neurosurg 24:726-730 
16. Lei W, Wu Z (2006) Biomechanical evaluation of an expansive pedicle screw in calf vertebrae. Eur Spine J 15:321-326

17. Ab-Lazid R, Perilli E, Ryan MK, Costi JJ, Reynolds KJ (2014) Does cancellous screw insertion torque depend on bone mineral density and/or microarchitecture? J Biomech 47:347-353

18. Inceoglu S, McLain RF, Cayli S, Kilincer C, Ferrara L (2004) Stress relaxation of bone significantly affects the pull-out behavior of pedicle screws. J Orthop Res 22:1243-1247

19. Nakashima D, Ishii K, Matsumoto M, Nakamura M, Nagura T (2018) A study on the use of the Osstell apparatus to evaluate pedicle screw stability: an in-vitro study using micro-CT. PLoS One 13:e0199362

20. Henyš P, Čapek L(2018) Impact force, polar gap and modal parameters predict acetabular cup fixation: a study on a composite bone. Ann Biomed Eng 46:590-604

21. Pastrav LC, Jaecques SV, Jonkers I, Perre GV, Mulier M (2009) In vivo evaluation of a vibration analysis technique for the per-operative monitoring of the fixation of hip prostheses. J Orthop Surg Res 4:10

22. Saha S, Lakes RS (1977) A non-invasive technique for detecting stress waves in bone using the piezoelectric effect. IEEE Trans Biomed Eng 24:508-512

23. Sennerby L, Meredith N (2008) Implant stability measurements using resonance frequency analysis: biological and biomechanical aspects and clinical implications. Periodontol 47:51-66

24. Valderrama P, Oates TW, Jones AA, Simpson J, Schoolfield JD, Cochran DL (2007) Evaluation of two different resonance frequency devices to detect implant stability: a clinical trial. J Periodontol 78:262-272

25. Panjabi MM, Krag M, Summers D, Videman T (1985) Biomechanical time-tolerance of fresh cadaveric human spine specimens. J Orthop Res 3:292-300

26. Matsukawa K, Yato Y, Kato T, Imabayashi H, Asazuma T, Nemoto K (2014) In vivo analysis of insertional torque during pedicle screwing using cortical bone trajectory technique. Spine (Phila Pa 1976) 39:E240-E245

27. Daftari TK, Horton WC, Hutton WC (1994) Correlations between screw hole preparation, torque of insertion, and pullout strength for spinal screws. J Spinal Disord 7:139-145

28. American Society for Testing and Materials (2007) ASTM F543-07, Standard specification and test methods for metallic medical bone screws, ASTM International, West Conshohocken, PA

29. Suzuki K, Miyakoshi N, Tsuchida T, Kasukawa Y, Sato K, Itoi E (2003) Effects of combined treatment of insulin and human parathyroid hormone(1-34) on cancellous bone mass and structure in streptozotocin-induced diabetic rats. Bone 33:108-114

30. Kazama JJ, Koda R, Yamamoto S, Narita I, Gejyo F, Tokumoto A (2009) Comparison of quantitative cancellous bone connectivity analyses at twoand three-dimensional levels in dialysis patients. Calcif Tissue Int 84:38-44

31. Hildebrand T, Rüegsegger P (1997) Quantification of bone microarchitecture with the structure model index. Comput Methods Biomech Biomed Engin $1: 15-23$

32. Tabor Z (2011) A novel method of estimating structure model index from gray-level images. Med Eng Phys 33:218-225

33. Coe JD, Warden KE, Herzig MA, McAfee PC (1990) Influence of bone mineral density on the fixation of thoracolumbar implants. A comparative study of transpedicular screws, laminar hooks, and spinous process wires. Spine (Phila Pa 1976) 15:902-907

34. Cho W, Wu C, Erkan S, Kang MM, Mehbod AA, Transfeldt EE (2011) The effect on the pullout strength by the timing of pedicle screw insertion after calcium phosphate cement injection. J Spinal Disord Tech 24:116-120

35. Cook SD, Salkeld SL, Stanley T, Faciane A, Miller SD (2004) Biomechanical study of pedicle screw fixation in severely osteoporotic bone. Spine J 4: 402-408

36. Siddiqui AA, Blakemore ME, Tarzi I (2005) Experimental analysis of screw hold as judged by operators $\vee$ pullout strength. Injury 36:55-59

37. Poukalova M, Yakacki CM, Guldberg RE et al (2010) Pullout strength of suture anchors: effect of mechanical properties of trabecular bone. Biomech 43:1138-1145

38. Yakacki CM, Poukalova M, Guldberg RE et al (2010) The effect of the trabecular microstructure on the pullout strength of suture anchors. Biomech 43:1953-1959

39. Mueller TL, Basler SE, Müller R, van Lenthe GH (2013) Time-lapsed imaging of implant fixation failure in human femoral heads. Med Eng Phys 35:636-643
40. Perilli E, Baruffaldi F, Bisi MC, Cristofolini L, Cappello A (2006) A physical phantom for the calibration of three-dimensional X-ray microtomography examination. J Microsc 222:124-134

41. Garrahan NJ, Mellish RW, Compston JE (1986) A new method for the twodimensional analysis of bone structure in human iliac crest biopsies. J Microsc 142:341-349

42. Yagi M, Ohne $\mathrm{H}$, Konomi T et al (2016) Teriparatide improves volumetric bone mineral density and fine bone structure in the UIV+1 vertebra, and reduces bone failure type PJK after surgery for adult spinal deformity. Osteoporos Int 27:3495-3502

43. Wichmann JL, Booz C, Wesarg S et al (2014) Quantitative dual-energy CT for phantomless evaluation of cancellous bone mineral density of the vertebral pedicle: correlation with pedicle screw pull-out strength. Eur Radiol. 25: $1714-1720$

\section{Submit your manuscript to a SpringerOpen ${ }^{\circ}$ journal and benefit from:}

- Convenient online submission

- Rigorous peer review

- Open access: articles freely available online

- High visibility within the field

- Retaining the copyright to your article

Submit your next manuscript at $\boldsymbol{\nabla}$ springeropen.com 